\title{
碱促进下碳二亚胺参与构建二氢喹唑啉及其衍生物
}

\author{
汤玲娟 $a, b$ 陆新谋 ${ }^{b}$ 纪顺俊*,b \\ ( ${ }^{a}$ 南通大学分析测试中心 南通 226019) \\ ( ${ }^{b}$ 江苏省有机合成重点实验室＼cjkstart苏州大学材料与化学化工学部＼cjkstart苏州 215123)
}

\begin{abstract}
摘要 发展了一种由含碳二亚胺结构的 $\alpha, \beta$-不饱和酮/酯和醇类化合物合成一系列具有潜在生理和药理活性的 2 -乙氧基 -3-苯基-3,4-二氢喹唑啉杂环化合物的方法. 反应条件温和、耗时短, 且为无金属催化反应, 反应收率最高可以达到 $99 \%$. 这为进一步构建喹唑啉这类含氮杂环化合物提供了一种有益策略.

关键词 碳二亚胺; $\alpha, \beta$-不饱和酮/酯; 喹唑啉; 无金属催化
\end{abstract}

\section{Base Promoted Annulation of Carbodiimides to Access of Quinazoline and Their Derivatives}

\author{
Tang, Lingjuan ${ }^{a, b} \quad$ Lu, Xinmou ${ }^{b} \quad$ Ji, Shunjun*,b \\ ( ${ }^{a}$ Analysis and Testing Center, Nantong University, Suzhou 226019) \\ $\left({ }^{b}\right.$ Key Laboratory of Organic Synthesis of Jiangsu Province, College of Chemistry, Chemical Engineering and \\ Materials Science, Soochow University, Suzhou 215123)
}

\begin{abstract}
An efficient methodology to access of quinazoline derivatives by the reaction of carbodiimides and alcohol under basic conditions has been developed. This paper provides an attractive strategy for construction of the quinazoline compounds and other heterocyclic compounds in a high reaction yield by using simple starting materials in mild reaction conditions and short reaction time.

Keywords carbodiimides; $\alpha, \beta$-unsaturated ketone; quinazoline and their derivatives; metal-free
\end{abstract}

二氢喹唑啉及其衍生物是许多精细化工产品的重 要中间体, 而且还具有多种生理功能和用途 ${ }^{[1]}$, 在医药 和工农业生产中有着广泛的应用. 如首个被分离得到的 含喹唑啉结构的化合物色胺酮 I, 抗菌效用明显. 随后 更多具有药用活性的含喹唑啉结构单元的化合物和类 似物相继被发现. 如利尿药甲酸喹唑酮 II、抗疮药常山 碱等 III. 此外, 喹唑啉类衍生物也被应用于农药开发方
面, 如杀螨剂喹螨醚 IV. 在工业领域, 喹唑啉类衍生物 可应用于有机发光材料 ${ }^{[2]}$; 在润滑剂、金属萃取炼等行 业也有广泛应用 ${ }^{[3]}$. 以上可知，喹唑啉衍生物在现代医 药和工农业生产中有着广泛的应用.

而传统合成含有喹唑啉骨架化合物的主要方法如 下(Scheme 1): 以邻氨基芐胺为底物 ${ }^{[4]}$ 、邻氨基/卤芳香 酮为底物 ${ }^{[5]}$ 的环化方法来合成是喹唑啉化合物. 以邻<smiles>O=C1c2ccccc2-n2c1nc1ccccc1c2=O</smiles>

I<smiles>Cc1ccccc1-n1c(CF)nc2ccccc2c1=O</smiles><smiles>O=C(CC1NCCC[C@H]1O)Cn1cnc2ccccc2c1=O</smiles>

III

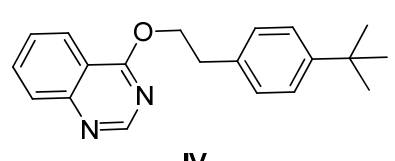

IV

图 1 具有生物活性的喹唑啉类化合物和其类似物

Figure 1 Select biologically active quinazoline and their derivatives

* Corresponding author. E-mail: shunjun@suda.edu.cn

Received July 5, 2016; revised October 19, 2016; published online December 12, 2016

Project supported by the National Natural Science Foundation of China (Nos. 21372174, 21542015), the Priority Academic Program Development of Jiangsu Higher Education Institutions.

国家自科学然基金(No. 21372174)、江苏省高校优势学科建设工程资助项目. 


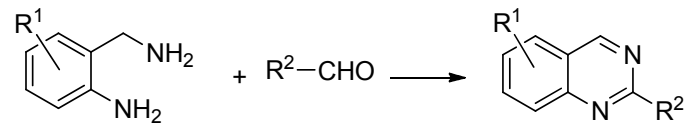

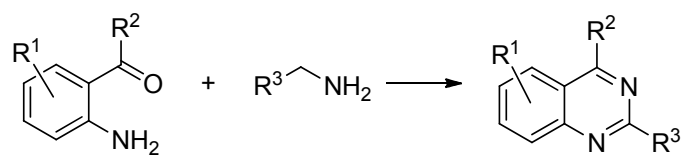<smiles>[R1]c1ccc(Cl)c(C(C)=O)c1</smiles>

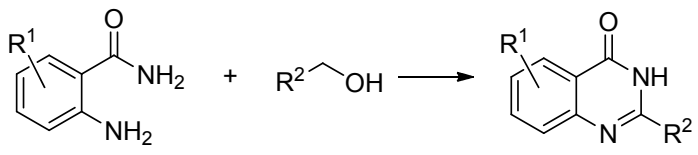<smiles></smiles><smiles>[R]C(=O)NC(=O)c1ccccc1N=Pc1ccccc1</smiles>

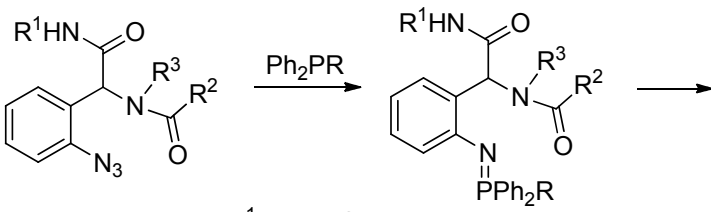<smiles>[R]NC(=O)C1c2ccccc2N=C([R])N1[R]</smiles>

图式 1 传统合成喹唑啉衍生物

Scheme 1 Traditional methods for the construction of quinazoline derivatives
氨基苯甲酰胺为底物 ${ }^{[6]}$ 、邻氨基苯甲酸为底物 ${ }^{[7]}$ ，以及利 用氮杂 Wittig 反应来合成喹唑啉酮及其衍生物 ${ }^{[8]}$. 而行 之有效地合成二氢喹唑啉的报道相对就比较少, 并且受 限于以下缺点: (1)反应条件较为苛刻; (2)原子经济性不 高、反应的步骤繁琐; (3)强碱和金属试剂的不同程度等.

近年来, 碳二亚胺化合物参与构建喹唑啉、喹唑啉 酮及其衍生物的研究获得了一系列报道. 有利用邻碘苯 胺、碳二亚胺和 $\mathrm{CO}$ 在钯盐催化合成 ${ }^{[9,10]}$, 二-邻碘苯基 碳二亚胺与有机胺化合物在钯盐催化合成 ${ }^{[10]}$, 以及 $\mathrm{Wu}$ 课题组 ${ }^{[11]}$ 利用碳二亚胺、烷基异氧和烷基胺/亚磷酸二 乙酯在钯盐催化合成(Scheme 2). 总结这些报道, 我们 可以看到反应均需要金属催化，有的反应甚至需要用到 易爆物质, 并且合成的都是喹唑啉酮类化合物, 更为重 要的二氢喹唑啉类化合物研究得较少.

2015 年我们课题组 ${ }^{[12]}$ 利用苯胺与异睛反应能以较 高收率得到相应的碳二亚胺化合物, 联系前人关于碳二 亚胺化合物参与构建二氢喹唑啉及其衍生物的报 道 ${ }^{[9 \sim 11]}$, 以及我们课题组前面关于吲哚的工作 ${ }^{[13]}$, 如果 对碳二亚胺化合物进行适当官能团修饰后，能否在碱作 用下与强亲核试剂吲哚反应并关环来构建一种含有喹 唑啉及吲哚官能团结构的化合物?

\section{1 结果与讨论}

基于以上设想，首先我们选取了 1-苯基-3-(2-(( 苯 基亚氨基)亚甲基)氨基)苯基)丙-2-烯-1-酮(1a)、 $N$-甲基 吲哚(2a), 在以无水乙醇为溶剂, 2 equiv. 碱作用下反应 以期生成含有吲哚取代基团的喹唑啉目标化合物 $\mathbf{3} \mathbf{a}^{\prime}$ (Scheme 3), 但从 LC-MS 以及核磁谱图结果来看并未生<smiles>[R][R]Oc1nc2ccccc2c(=N[R])n1[R]</smiles>

图式 2 碳二亚胺化合物参与构建喹唑啉衍生物

Scheme 2 Construction of quinazoline derivatives via carbodiimide 


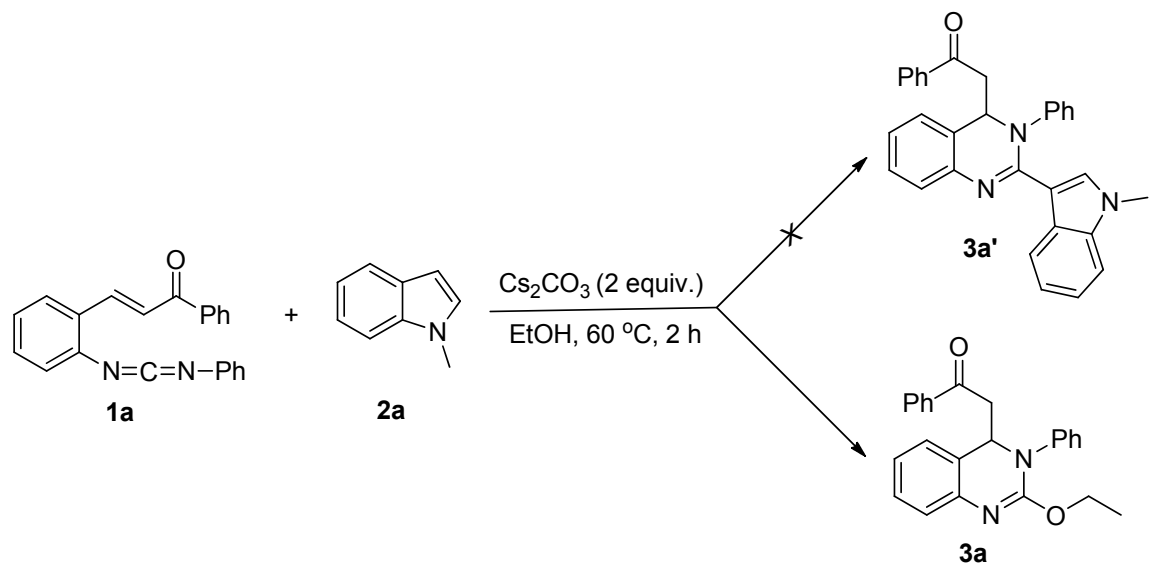

图式 3 喹唑啉衍生物合成

Scheme 3 Construction of quinazoline derivatives

成预想的目标化合物. 为进一步验证产物结构, 以乙酸 乙酯为溶剂培养了化合物单晶确证了化合物 $\mathbf{3 a}$ 的结构 (图 2), CCDC 号为 1510457 .

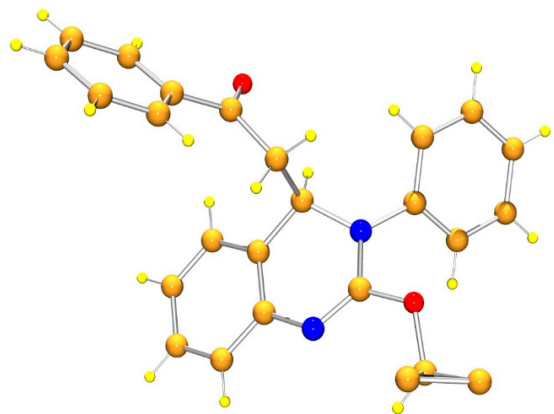

图 2 化合物 3a 的单晶结构

Figure 2 X-ray structure of $\mathbf{3 a}$

对该反应的条件优化结果见表 1. 首先我们发现在 不加碱情况下反应不能发生(Table 1, Entry 1), 然后对 各种不同的无机碱进行了篮选, 发现当使用无机弱碱, 如 $\mathrm{Cs}_{2} \mathrm{CO}_{3} 、 \mathrm{~K}_{2} \mathrm{CO}_{3} 、 \mathrm{Na}_{2} \mathrm{CO}_{3} 、 \mathrm{NaHCO}_{3} 、 \mathrm{KHCO}_{3}$ 时, 液 质收率均在 $60 \% \sim 70 \%$ 之间(Table 1, Entries 2６). 接着 改用 $\mathrm{KOH} 、 \mathrm{NaOH} 、 t$-BuOK 进行反应时, 反应液质收率 提升至 $70 \%$ 以上，其中以使用 $\mathrm{NaOH}$ 为碱时液质收率最 高(77\%) (Table 1, Entries 7 9). 之后对几种有机碱进行 了篮选, 发现反应效果均不如 $\mathrm{NaOH}$ 好(Table 1, Entries 10 13). 篮完碱后, 我们对反应时间进行了优化, 反应 时间为 $1.5 \mathrm{~h}$ 时液质收率最高(Table 1, Entries 14 19). 接着对反应温度进行了䇻选, 发现 $40{ }^{\circ} \mathrm{C}$ 时反应效果最 好(Table 1, Entries 20 23). 对反应所用碱 $\mathrm{NaOH}$ 的用 量进行了篮选, 得出 1 equiv. $\mathrm{NaOH}$ 用量为最佳用量, $\mathbf{3 a}$ 分离收率高达 84\%(Table 1, Entries 24 28). 综合分析 以上数据, 我们得出 $1 \mathrm{a}(0.5 \mathrm{mmol})$ 在 1 equiv. $\mathrm{NaOH}$ 碱 存在下在 $2 \mathrm{~mL} \mathrm{EtOH}$ 中于 $40{ }^{\circ} \mathrm{C}$ 下反应 $1.5 \mathrm{~h}$ 为最优反 应条件。
表 1 条件篮选

Table 1 Optimization of reaction conditions

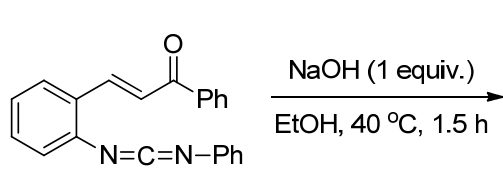

1a<smiles>CCOC1=Nc2ccccc2C(CC(=O)c2ccccc2)N1c1ccccc1</smiles>

3a

\begin{tabular}{|c|c|c|c|c|}
\hline Entry & Base (equiv.) & $T^{b} /{ }^{\circ} \mathrm{C}$ & Time/h & Yield ${ }^{c} / \%$ \\
\hline 1 & - & 40 & 1.5 & NR \\
\hline 2 & $\mathrm{Cs}_{2} \mathrm{CO}_{3}(2)$ & 40 & 1.5 & 67 \\
\hline 3 & $\mathrm{~K}_{2} \mathrm{CO}_{3}(2)$ & 40 & 1.5 & 69 \\
\hline 4 & $\mathrm{Na}_{2} \mathrm{CO}_{3}(2)$ & 40 & 1.5 & 63 \\
\hline 5 & $\mathrm{NaHCO}_{3}(2)$ & 40 & 1.5 & 64 \\
\hline 6 & $\mathrm{KHCO}_{3}(2)$ & 40 & 1.5 & 69 \\
\hline 7 & $\mathrm{KOH}(2)$ & 40 & 1.5 & 74 \\
\hline 8 & $\mathrm{NaOH}(2)$ & 40 & 1.5 & 77 \\
\hline 9 & $t$-BuOK (2) & 40 & 1.5 & 71 \\
\hline 10 & Pyridine (2) & 40 & 1.5 & 55 \\
\hline 11 & Piperidine (2) & 40 & 1.5 & NR \\
\hline 12 & $\mathrm{Et}_{3} \mathrm{~N}(2)$ & 40 & 1.5 & 59 \\
\hline 13 & DBU (2) & 40 & 1.5 & NR \\
\hline 14 & $\mathrm{NaOH}(2)$ & 40 & 0.25 & 45 \\
\hline 15 & $\mathrm{NaOH}(2)$ & 40 & 0.5 & 58 \\
\hline 16 & $\mathrm{NaOH}(2)$ & 40 & 1 & 61 \\
\hline 17 & $\mathrm{NaOH}(2)$ & 40 & 1.5 & 77 \\
\hline 18 & $\mathrm{NaOH}(2)$ & 40 & 2 & 61 \\
\hline 19 & $\mathrm{NaOH}(2)$ & 40 & 4 & 59 \\
\hline 20 & $\mathrm{NaOH}(2)$ & 20 & 1.5 & 52 \\
\hline 21 & $\mathrm{NaOH}(2)$ & 40 & 1.5 & 75 \\
\hline 22 & $\mathrm{NaOH}(2)$ & 60 & 1.5 & 77 \\
\hline 23 & $\mathrm{NaOH}(2)$ & Reflux & 1.5 & 73 \\
\hline 26 & $\mathrm{NaOH}(0.5)$ & 40 & 1.5 & 70 \\
\hline 27 & $\mathrm{NaOH}(1)$ & 40 & 1.5 & $90(84)^{d}$ \\
\hline 28 & $\mathrm{NaOH}(1.5)$ & 40 & 1.5 & 87 \\
\hline
\end{tabular}

${ }^{a}$ Reaction conditions: 1a $(0.5 \mathrm{mmol})$ reacted in $2.0 \mathrm{~mL}$ of EtOH. ${ }^{b}$ The temperature of the reaction in solvent. ${ }^{c}$ Yields were determined by LC analysis with biphenyl as the internal standard. ${ }^{d}$ Isolated yield. 
表 2 喹唑啉衍生物 $\mathbf{3}$ 的合成

Table 2 Synthesis of 2-ethoxy-3,4-dihydroquinazolines 3

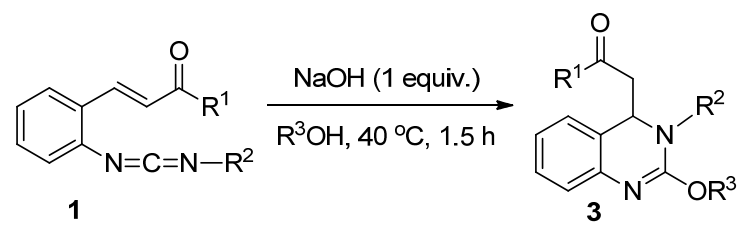<smiles>CCOC1=Nc2ccccc2C(CC(=O)c2ccccc2)N1c1ccccc1</smiles><smiles>COC1=Nc2ccccc2C(CC(=O)c2ccccc2)N1c1ccccc1</smiles>

3b, $85 \%$

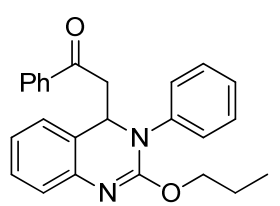

3c, $74 \%$<smiles>CCCCOC1=Nc2ccccc2C(CC(=O)c2ccccc2)N1c1ccccc1</smiles><smiles>CC(C)OC1=Nc2ccccc2C(CC(=O)c2ccccc2)N1c1ccccc1</smiles><smiles>CCCCOC(=O)CC1c2ccccc2N=C(OCC)N1c1ccc(Br)cc1</smiles>

$3 g, 85 \%$
$3 e, 60 \%$<smiles>CCOC1=Nc2ccccc2C(CC(=O)c2ccccc2)N1c1ccc(OC)cc1</smiles>

3h, $86 \%$

3f, $99 \%$<smiles>CCOC1=Nc2ccccc2C(CC(=O)c2ccccc2)N1c1ccc(F)cc1</smiles>

的拓展研究, 发现苯环上无论含有拉电子基团还是供电 子基团，反应分离收率都能达到 $80 \%$ 以上(3f， 3g，3h). 值得注意的是当苯环上含有强拉电子基团 $(\mathrm{F})$ 时，反应 分离收率能达到 $99 \%$ 以上(3f)，几乎定量生成目标化合 物.

接着我们又对 3-(2-((苯基亚氨基)亚甲基)氨基)苯 基)丙烯酸酯底物进行了拓展研究(表 3), 如所预期的那 样，在前面最优条件下，反应能生成相应的喹唑啉目标 化合物，但是在碱性条件下，也发生了相应的酯交换反 应. 对 $\mathrm{R}^{1}$ 含不同取代基团时进行了底物的拓展研究，发 现 $\mathrm{R}^{1}$ 为无取代苯环基团时，分离收率为 79\%(4b)，而当 $\mathrm{R}^{1}$ 苯环上含有供电子基团 $\left(\mathrm{OCH}_{3}\right)$, 反应分离收率为 $80 \%(4 a)$, 而当 $\mathrm{R}^{1}$ 环己基时，反应分离收率则下降至 $74 \%(4 c)$

表 3 喹唑啉衍生物 $\mathbf{4}$ 的合成

Table 3 Synthesis of $2 H$-pyrrolo[3,4-c] quinolin-4(5H)-ones 4

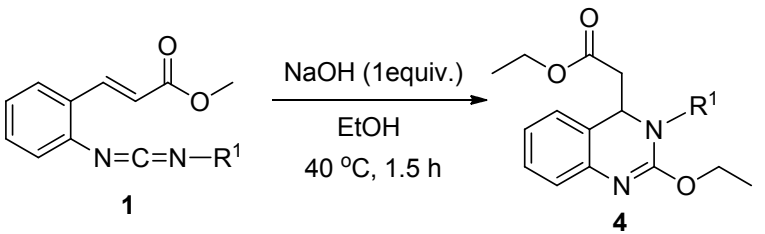<smiles>CCOC(=O)CC1c2ccccc2N=C(OCC)N1c1cccc(OCC)c1</smiles>

$4 a, 80 \%$<smiles>CCOC(=O)CC1c2ccccc2N=C(OCC)N1c1ccccc1</smiles>

$4 \mathbf{b}, 79 \%$<smiles>CCOC(=O)CC1c2ccccc2N=C(OCC)N1C1CCCCC1</smiles>

4c, $74 \%$
${ }^{a}$ Reaction conditions: $1(0.5 \mathrm{mmol})$ reacted in ethanol at $40{ }^{\circ} \mathrm{C}$ for $1.5 \mathrm{~h}$. ${ }^{b}$ Isolated yield.

基于以上结果及查阅相关文献 [8], 我们提出了一 个可能的反应机理, 如 Scheme 4 所示. 首先是醇在碱作 用下生成醇盐负离子 $\mathbf{A}$, 醇盐负离子 $\mathbf{A}$ 对碳二亚胺化合 因，接着我们对 $\mathrm{R}^{2}$ 含不同取代基团时进行了底物

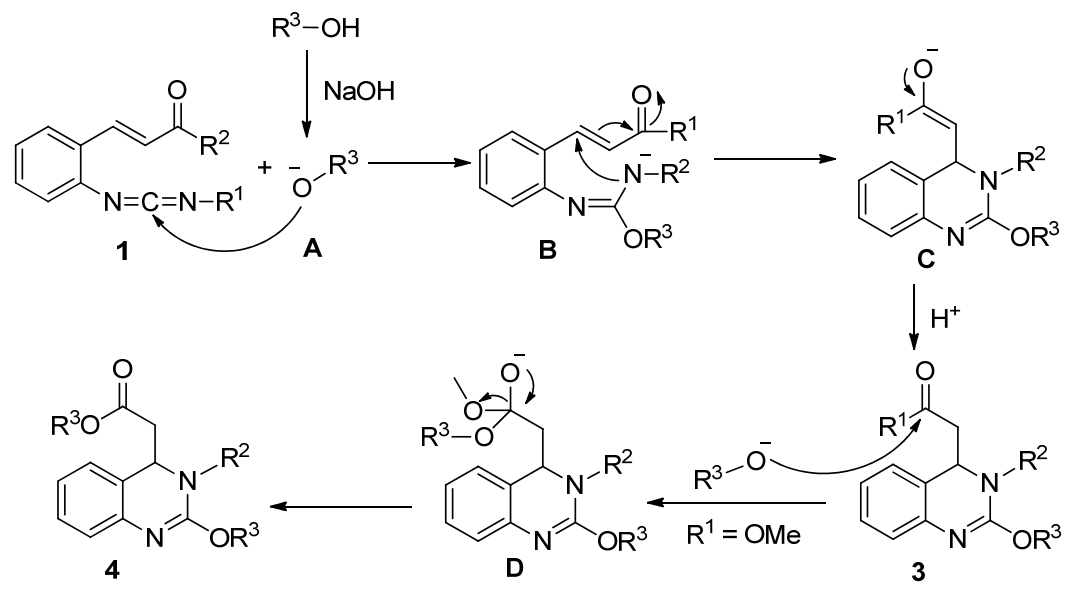

图式 4 可能的反应机理

Scheme 4 A plausible mechanism 
物发动亲核进攻生成胍中间体 $\mathbf{B}$, 之后胍中间体 $\mathbf{B}$ 发生 分子内麦克加成生成中间体 $\mathbf{C}$, 最后 $\mathbf{C}$ 烯醇互变生成产 物 $\mathbf{3}$, 当 $\mathrm{R}^{1}=\mathrm{OMe}$ 时, 产物 $\mathbf{3}$ 发生酯交换生成最终产物 4.

\section{2 结论}

发展了一种含碳二亚胺结构的 $\alpha, \beta$-不饱和酩/酯与 醇类化合物反应生成一系列具有潜在生理和药理活性 的 2-乙氧基-3-苯基-3,4-二氢喹唑啉杂环衍生物的方法. 反应在无金属催化条件下进行, 反应条件温和, 反应耗 时短, 反应收率高达 $99 \%$. 这为进一步构建这类喹唑啉 化合物以及其他的杂环化合物提供了一种有益策略.

\section{3 实验部分}

\section{1 仪器与试剂}

核磁共振 Varian InoVa-400 MHz 或 Varian NMR System $300 \mathrm{MHz}$ 检测完成, 以 $\mathrm{CDCl}_{3}$ 为溶剂, TMS 为内 标; 红外光谱由 Varian FT-1000 红外光谱仪, KBr 压片 完成; 化合物的熔点由 X-T5 型熔点测定仪, 温度计未 校正. 其他试剂均为市售分析纯.

\section{2 实验方法}

化合物 $1(0.5 \mathrm{mmol}) 、 \mathrm{NaOH}(0.5 \mathrm{mmol})$ 以及 $3.0 \mathrm{~mL}$ $\mathrm{ROH}$ 置于 Schlenk 反应管中, 油浴加热至 $40{ }^{\circ} \mathrm{C}$ 搅拌反 应 $1.5 \mathrm{~h}$. 反应结束后减压旋蒸除去反应溶剂, 进一步柱 层析 $[V($ 乙酸乙酯 $) / V$ (石油醚 $)=1 / 10]$ 得目标化合物 $2,4-$ 二取代喹唑啉类衍生物.

2-(2-乙氧基-3-苯基-3,4-二氢喹唑啉-4-基)-1-苯丙酮 (3a): 黄色油状物. ${ }^{1} \mathrm{H}$ NMR $\left(400 \mathrm{MHz}, \mathrm{CDCl}_{3}\right) \delta$ : 7.78 $7.69(\mathrm{~m}, 2 \mathrm{H}), 7.48 \sim 7.39(\mathrm{~m}, 1 \mathrm{H}), 7.35 \sim 7.25(\mathrm{~m}, 4 \mathrm{H})$, $7.24 \sim 7.06(\mathrm{~m}, 6 \mathrm{H}), 6.89$ (ddd, $J=7.6,5.4,3.2 \mathrm{~Hz}, 1 \mathrm{H}$ ), $5.54(\mathrm{dd}, J=8.7,4.0 \mathrm{~Hz}, 1 \mathrm{H}), 4.43(\mathrm{q}, J=7.1 \mathrm{~Hz}, 2 \mathrm{H})$, 3.59 (dd, $J=16.2,8.7 \mathrm{~Hz}, 1 \mathrm{H}), 3.33$ (dd, $J=16.2,4.0 \mathrm{~Hz}$, $1 \mathrm{H}), 1.26(\mathrm{t}, J=7.1 \mathrm{~Hz}, 3 \mathrm{H}$ ); IR (neat) $v: 3065,2932$, $1737,1585,1553,1471,1445,766,754 \mathrm{~cm}^{-1} ;{ }^{13} \mathrm{C}$ NMR $\left(101 \mathrm{MHz}\right.$, DMSO- $\left.d_{6}\right) \delta: 197.8,153.4,146.6,142.3,137.1$, $133.8,130.6,129.4,129.4,129.1,128.5,126.0,125.9$, 125.7, 125.6, 123.3, 123.1, 63.0, 59.2, 44.4, 14.6. HRMS (ESI-TOF) calcd for $\mathrm{C}_{24} \mathrm{H}_{23} \mathrm{~N}_{2} \mathrm{O}_{2}\left([\mathrm{M}+\mathrm{H}]^{+}\right) 371.1760$, found 371.1758.

2-(2-甲氧基-3-苯基-3,4-二氢喹唑啉-4-基)-1-苯丙酮 (3b): 黄色固体, m.p. $57 \sim 59{ }^{\circ} \mathrm{C} ;{ }^{1} \mathrm{H}$ NMR $(400 \mathrm{MHz}$, $\left.\mathrm{CDCl}_{3}\right) \delta: 7.74 \sim 7.69(\mathrm{~m}, 2 \mathrm{H}), 7.45 \sim 7.40(\mathrm{~m}, 1 \mathrm{H})$, $7.32 \sim 7.26(\mathrm{~m}, 4 \mathrm{H}), 7.23 \sim 7.14(\mathrm{~m}, 5 \mathrm{H}), 7.10 \sim 7.07(\mathrm{~m}$, $1 \mathrm{H}), 6.89$ (ddd, $J=7.6,6.1,2.6 \mathrm{~Hz}, 1 \mathrm{H}), 5.54(\mathrm{dd}, J=8.6$, $4.0 \mathrm{~Hz}, 1 \mathrm{H}), 3.90$ (s, 3H), 3.57 (dd, $J=16.2,8.7 \mathrm{~Hz}, 1 \mathrm{H}$ ), $3.32(\mathrm{dd}, J=16.2,4.0 \mathrm{~Hz}, 1 \mathrm{H}) ;{ }^{13} \mathrm{C}$ NMR $(101 \mathrm{MHz}$, $\left.\mathrm{CDCl}_{3}\right) \delta: 197.3,154.1,142.4,141.8,136.9,133.2,129.0$, 128.4, 128.1, 125.9, 125.6, 125.56, 125.4, 123.4, 123.1, 59.2, 54.3, 44.1; IR (neat) v: 3063, 2939, 1735, 1586, $1559,1472,1441,761,758 \mathrm{~cm}^{-1}$. HRMS (ESI-TOF) calcd for $\mathrm{C}_{23} \mathrm{H}_{21} \mathrm{~N}_{2} \mathrm{O}_{2}\left([\mathrm{M}+\mathrm{H}]^{+}\right)$357.1603, found 357.1590.

2-(2-丙氧基-3-苯基-3,4-二氢喹唑啉-4-基)-1-苯丙酮 (3c): 黄色油状物. ${ }^{1} \mathrm{H}$ NMR ( $\left.400 \mathrm{MHz}, \mathrm{CDCl}_{3}\right) \delta$ : 7.77 $7.71(\mathrm{~m}, 2 \mathrm{H}), 7.46(\mathrm{t}, J=7.4 \mathrm{~Hz}, 1 \mathrm{H}), 7.36 \sim 7.28(\mathrm{~m}, 4 \mathrm{H})$, $7.25 \sim 7.14$ (m, 5H), $7.08(\mathrm{~d}, J=7.4 \mathrm{~Hz}, 1 \mathrm{H}), 6.94 \sim 6.87$ (m, 1H), 5.54 (dd, $J=8.7,4.0 \mathrm{~Hz}, 1 \mathrm{H}), 4.31$ (tt, $J=7.8,3.8$ $\mathrm{Hz}, 2 \mathrm{H}), 3.59$ (dd, $J=16.1,8.7 \mathrm{~Hz}, 1 \mathrm{H}), 3.34$ (dd, $J=16.1$, $4.1 \mathrm{~Hz}, 1 \mathrm{H}), 1.68 \sim 1.60(\mathrm{~m}, 2 \mathrm{H}), 0.86(\mathrm{t}, J=7.4 \mathrm{~Hz}, 3 \mathrm{H})$; ${ }^{13} \mathrm{C} \mathrm{NMR}\left(101 \mathrm{MHz}, \mathrm{CDCl}_{3}\right) \delta: 197.6,153.9,133.3,128.9$, $128.6,128.5,128.2,125.8,125.7,125.6,125.5,123.3$, 123.1, 68.9, 59.2, 44.2, 22.2, 10.6; IR (neat) $v: 3069,2935$, 1732, 1584, 1558, 1472, 1441, 769, $751 \mathrm{~cm}^{-1}$. HRMS (ESI-TOF) calcd for $\mathrm{C}_{25} \mathrm{H}_{25} \mathrm{~N}_{2} \mathrm{O}_{2}\left([\mathrm{M}+\mathrm{H}]^{+}\right) 385.1916$, found 385.1904 .

2-(2-丁氧基-3-苯基-3,4-二氢喹唑啉-4-基)-1-苯丙酮 (3d): 黄色固体, m.p. $80 \sim 82{ }^{\circ} \mathrm{C} ;{ }^{1} \mathrm{H}$ NMR (400 MHz, $\left.\mathrm{CDCl}_{3}\right) \delta: 7.80 \sim 7.70(\mathrm{~m}, 2 \mathrm{H}), 7.53 \sim 7.44(\mathrm{~m}, 1 \mathrm{H})$, $7.38 \sim 7.27(\mathrm{~m}, 4 \mathrm{H}), 7.24 \sim 7.13(\mathrm{~m}, 5 \mathrm{H}), 7.08(\mathrm{dd}, J=7.6$, $1.3 \mathrm{~Hz}, 1 \mathrm{H}), 6.95 \sim 6.86(\mathrm{~m}, 1 \mathrm{H}), 5.54(\mathrm{dd}, J=8.7,4.0 \mathrm{~Hz}$, $1 \mathrm{H}), 4.36(\mathrm{t}, J=6.6 \mathrm{~Hz}, 2 \mathrm{H}), 3.60(\mathrm{dd}, J=16.1,8.7 \mathrm{~Hz}$, $1 \mathrm{H}), 3.34(\mathrm{dd}, J=16.1,4.1 \mathrm{~Hz}, 1 \mathrm{H}), 1.67 \sim 1.57(\mathrm{~m}, 2 \mathrm{H})$, $1.34 \sim 1.27(\mathrm{~m}, 2 \mathrm{H}), 0.88(\mathrm{t}, J=7.4 \mathrm{~Hz}, 3 \mathrm{H}) ;{ }^{13} \mathrm{C} \mathrm{NMR}$ $\left(101 \mathrm{MHz}, \mathrm{CDCl}_{3}\right) \delta: 197.0,153.3,142.1,141.5,136.5$, $132.7,128.4,128.0,127.9,127.7,125.2,125.2,125.0$, 124.9, 122.7, 122.6, 66.6, 58.7, 43.6, 30.3, 18.7, 13.3; IR (neat) $v: 3061,2933,1731,1589,1555,1474,1447,761$, $755 \mathrm{~cm}^{-1}$. HRMS (ESI-TOF) calcd for $\mathrm{C}_{26} \mathrm{H}_{27} \mathrm{~N}_{2} \mathrm{O}_{2}$ ([M+ $\mathrm{H}]^{+}$) 399.2073, found 399.2070 .

2-(2-异丙氧基-3-苯基-3,4-二氢喹唑啉-4-基)-1-苯丙 酮(3e): 黄色固体, m.p 120 122 ${ }^{\circ} \mathrm{C} ;{ }^{1} \mathrm{H}$ NMR (400 $\left.\mathrm{MHz}, \mathrm{CDCl}_{3}\right) \delta: 7.76(\mathrm{~d}, J=7.3 \mathrm{~Hz}, 2 \mathrm{H}), 7.49$ (t, $J=7.4$ $\mathrm{Hz}, 1 \mathrm{H}), 7.38 \sim 7.28(\mathrm{~m}, 4 \mathrm{H}), 7.23 \sim 7.12(\mathrm{~m}, 5 \mathrm{H}), 7.08(\mathrm{~d}$, $J=7.3 \mathrm{~Hz}, 1 \mathrm{H}), 6.90(\mathrm{td}, J=7.2,1.4 \mathrm{~Hz}, 1 \mathrm{H}), 5.53(\mathrm{dd}$, $J=8.7,4.0 \mathrm{~Hz}, 1 \mathrm{H}), 5.42(\mathrm{dt}, J=12.5,6.2 \mathrm{~Hz}, 1 \mathrm{H}), 3.59$ (dd, $J=16.1,8.7 \mathrm{~Hz}, 1 \mathrm{H}), 3.34$ (dd, $J=16.1,4.0 \mathrm{~Hz}, 1 \mathrm{H}$ ), $1.33(\mathrm{~d}, J=6.3 \mathrm{~Hz}, 3 \mathrm{H}), 1.23(\mathrm{~d}, J=6.2 \mathrm{~Hz}, 3 \mathrm{H}) ;{ }^{13} \mathrm{C}$ NMR (101 MHz, $\left.\mathrm{CDCl}_{3}\right) \delta$ : 197.2, 152.6, 142.3, 141.67, $136.6,132.7,128.3,128.0,127.9,127.7,125.3,124.9$, 124.7, 122.6, 122.5, 69.7, 58.6, 43.5, 21.7, 21.1; IR (neat) $v: 3072,2947,1738,1579,1566,1466,1453,774,756$ 
$\mathrm{cm}^{-1}$. HRMS (ESI-TOF) calcd for $\mathrm{C}_{25} \mathrm{H}_{25} \mathrm{~N}_{2} \mathrm{O}_{2}\left([\mathrm{M}+\mathrm{H}]^{+}\right)$ 385.1916, found 385.1919.

2-(2-乙氧基-3-对氟苯基-3,4-二氢喹唑啉-4-基)-1-苯 丙酮(3f): 黄色固体, m.p. 92 93 ${ }^{\circ} \mathrm{C} ;{ }^{1} \mathrm{H}$ NMR (400 $\left.\mathrm{MHz}, \mathrm{CDCl}_{3}\right) \delta: 7.75 \sim 7.67(\mathrm{~m}, 2 \mathrm{H}), 7.47 \sim 7.40(\mathrm{~m}, 1 \mathrm{H})$, $7.31(\mathrm{dd}, J=8.3,7.2 \mathrm{~Hz}, 2 \mathrm{H}), 7.23 \sim 7.13(\mathrm{~m}, 4 \mathrm{H}), 7.09 \sim$ $7.04(\mathrm{~m}, 1 \mathrm{H}), 7.02 \sim 6.94(\mathrm{~m}, 2 \mathrm{H}), 6.90(\mathrm{ddd}, J=7.5,6.2$, $2.4 \mathrm{~Hz}, 1 \mathrm{H}), 5.48$ (dd, $J=8.1,4.4 \mathrm{~Hz}, 1 \mathrm{H}), 4.40$ (qd, $J=$ 7.1, $5.2 \mathrm{~Hz}, 2 \mathrm{H}), 3.50$ (dd, $J=16.1,8.1 \mathrm{~Hz}, 1 \mathrm{H}), 3.33$ (dd, $J=16.1,4.4 \mathrm{~Hz}, 1 \mathrm{H}), 1.23$ (t, $J=7.1 \mathrm{~Hz}, 3 \mathrm{H}) ;{ }^{13} \mathrm{C} \mathrm{NMR}$ $\left(101 \mathrm{MHz}, \mathrm{CDCl}_{3}\right) \delta: 197.3,153.4,142.6,138.0,137.9$, $136.9,133.2,128.5,128.1,127.8,127.7,125.4,125.3$, $123.3,123.1,115.8,115.5,63.1,59.6,44.1,14.3$; IR (neat) $v: 3064,2936,1671,1585,1568,1475,752,744 \mathrm{~cm}^{-1}$. HRMS (ESI-TOF) calcd for $\mathrm{C}_{24} \mathrm{H}_{22} \mathrm{FN}_{2} \mathrm{O}_{2}\left([\mathrm{M}+\mathrm{H}]^{+}\right)$ 389.1665, found 389.1671 .

2-(2-乙氧基-3-对溴苯基-3,4-二氢喹唑啉-4-基)-1-苯 丙酩(3g): 黄色固体, m.p. 108 109 ${ }^{\circ} \mathrm{C} ;{ }^{1} \mathrm{H}$ NMR (400 $\left.\mathrm{MHz}, \mathrm{CDCl}_{3}\right) \delta: 7.79 \sim 7.66(\mathrm{~m}, 2 \mathrm{H}), 7.45$ (ddt, $J=8.8$, 6.9, $1.6 \mathrm{~Hz}, 1 \mathrm{H}), 7.42 \sim 7.36(\mathrm{~m}, 2 \mathrm{H}), 7.32(\mathrm{t}, J=7.8 \mathrm{~Hz}$, $2 \mathrm{H}), 7.23 \sim 7.14(\mathrm{~m}, 2 \mathrm{H}), 7.13 \sim 7.03(\mathrm{~m}, 3 \mathrm{H}), 6.91$ (ddd, $J=7.5,6.2,2.4 \mathrm{~Hz}, 1 \mathrm{H}), 5.50$ (dd, $J=8.3,4.3 \mathrm{~Hz}, 1 \mathrm{H})$, 4.42 (q, $J=6.6,6.2 \mathrm{~Hz}, 2 \mathrm{H}), 3.52$ (dd, $J=16.3,8.3 \mathrm{~Hz}$, $1 \mathrm{H}), 3.31$ (dd, $J=16.2,4.3 \mathrm{~Hz}, 1 \mathrm{H}), 1.26(\mathrm{t}, J=7.1 \mathrm{~Hz}$, $3 \mathrm{H}) ;{ }^{13} \mathrm{C}$ NMR (101 MHz, $\left.\mathrm{CDCl}_{3}\right) \delta: 197.3,153.1,142.3$, $141.0,136.8,133.3,131.9,128.5,128.5,128.1,126.7$, 125.6, 125.3, 123.5, 123.2, 118.7, 63.2, 59.0, 44.0, 14.3; IR (neat) $v: 3068,2937,1734,1582,1556,1476,1443,764$, $755 \mathrm{~cm}^{-1}$. HRMS (ESI-TOF) calcd for $\mathrm{C}_{24} \mathrm{H}_{22} \mathrm{BrN}_{2} \mathrm{O}_{2}$ $\left([\mathrm{M}+\mathrm{H}]^{+}\right) 449.0865$, found 449.0863 .

2-(2-乙氧基-3-对甲氧基苯基-3,4-二氢喹唑啉-4基)-1-苯丙酮(3h): 黄色固体, m.p. 57 59 ${ }^{\circ} \mathrm{C} ;{ }^{1} \mathrm{H} \mathrm{NMR}$ $\left(400 \mathrm{MHz}, \mathrm{CDCl}_{3}\right) \delta: 7.70(\mathrm{dd}, J=8.3,1.4 \mathrm{~Hz}, 2 \mathrm{H})$, $7.45 \sim 7.38(\mathrm{~m}, 1 \mathrm{H}), 7.29(\mathrm{t}, J=7.8 \mathrm{~Hz}, 2 \mathrm{H}), 7.21 \sim 7.09$ $(\mathrm{m}, 4 \mathrm{H}), 7.05(\mathrm{~d}, J=7.0 \mathrm{~Hz}, 1 \mathrm{H}), 6.94 \sim 6.74(\mathrm{~m}, 3 \mathrm{H})$, $5.45(\mathrm{dd}, J=8.4,4.2 \mathrm{~Hz}, 1 \mathrm{H}), 4.46 \sim 4.32(\mathrm{~m}, 2 \mathrm{H}), 3.73(\mathrm{~d}$, $J=1.3 \mathrm{~Hz}, 3 \mathrm{H}), 3.53(\mathrm{dd}, J=15.9,8.4 \mathrm{~Hz}, 1 \mathrm{H}), 3.32(\mathrm{dd}, J$ $=15.9,4.2 \mathrm{~Hz}, 1 \mathrm{H}), 1.22(\mathrm{t}, J=7.1 \mathrm{~Hz}, 3 \mathrm{H}) ;{ }^{13} \mathrm{C} \mathrm{NMR}$ $\left(101 \mathrm{MHz}, \mathrm{CDCl}_{3}\right) \delta: 197.4,157.6,153.9,142.9,136.9$, $134.7,133.1,128.4,128.3,128.1,127.7,125.3,125.3$, 123.0, 122.9, 114.1, 62.9, 59.8, 55.3, 44.1, 14.4; IR (neat) $v$ : 3062, 3030, 2992, 2937, 1733, 1583, 1566, 770, 755, $741 \mathrm{~cm}^{-1}$. HRMS (ESI-TOF) calcd for $\mathrm{C}_{25} \mathrm{H}_{25} \mathrm{~N}_{2} \mathrm{O}_{3}([\mathrm{M}+$ $\mathrm{H}]^{+}$) 401.1865 , found 401.1854 .

乙基-2-(2-乙氧基-3-(3-甲氧基苯基)-3,4-二氢喹唑 啉-4-基)乙酸酯(4a): 黄色油状物. ${ }^{1} \mathrm{H}$ NMR (400 MHz, $\left.\mathrm{CDCl}_{3}\right) \delta: 7.70(\mathrm{dd}, J=8.3,1.4 \mathrm{~Hz}, 4 \mathrm{H}), 7.47 \sim 7.37(\mathrm{~m}$, 2H), 7.29 (t, $J=7.8 \mathrm{~Hz}, 4 \mathrm{H}), 7.15$ (d, $J=6.4 \mathrm{~Hz}, 7 \mathrm{H}), 7.12$ $(\mathrm{s}, 1 \mathrm{H}), 7.05(\mathrm{~d}, J=7.0 \mathrm{~Hz}, 2 \mathrm{H}), 6.91 \sim 6.78(\mathrm{~m}, 6 \mathrm{H}), 5.45$ $(\mathrm{dd}, J=8.4,4.2 \mathrm{~Hz}, 2 \mathrm{H}), 4.46 \sim 4.32(\mathrm{~m}, 4 \mathrm{H}), 3.73(\mathrm{~d}, J=$ $1.3 \mathrm{~Hz}, 6 \mathrm{H}), 3.53$ (dd, $J=15.9,8.4 \mathrm{~Hz}, 2 \mathrm{H}), 3.32$ (dd, $J=$ 15.9, 4.2 Hz, 2H), 1.22 (t, $J=7.1 \mathrm{~Hz}, 7 \mathrm{H}) ;{ }^{13} \mathrm{C}$ NMR (101 $\left.\mathrm{MHz}, \mathrm{CDCl}_{3}\right) \delta: 197.4,157.6,153.9,142.9,136.9,134.7$, $133.1,128.4,128.3,128.1,127.7,125.3,125.3,123.0$, 122.9, 114.1, 62.9, 59.8, 55.3, 44.1, 14.4; IR (neat) v: 3066, 2936, 1730, 1587, 1566, 1475, $760 \mathrm{~cm}^{-1}$. HRMS (ESITOF) calcd for $\mathrm{C}_{21} \mathrm{H}_{25} \mathrm{~N}_{2} \mathrm{O}_{4}\left([\mathrm{M}+\mathrm{H}]^{+}\right)$369.1814, found 369.1811 .

乙基-2-(2-乙氧基-3-(3-苯基)-3,4-二氢喹唑啉-4-基) 乙酸酯(4c): 黄色油状物. ${ }^{1} \mathrm{H}$ NMR $\left(400 \mathrm{MHz}, \mathrm{CDCl}_{3}\right) \delta$ : $7.38 \sim 7.31(\mathrm{~m}, 2 \mathrm{H}), 7.28 \sim 7.17(\mathrm{~m}, 4 \mathrm{H}), 7.17 \sim 7.09(\mathrm{~m}$, $1 \mathrm{H}), 7.00$ (dtd, $J=8.6,7.5,1.4 \mathrm{~Hz}, 2 \mathrm{H}), 5.26$ (dd, $J=7.7$, $5.4 \mathrm{~Hz}, 1 \mathrm{H}), 4.48 \sim 4.27(\mathrm{~m}, 2 \mathrm{H}), 3.98$ (qq, $J=10.8,7.1$ $\mathrm{Hz}, 2 \mathrm{H}), 2.74$ (ddd, $J=22.5,14.7,6.6 \mathrm{~Hz}, 2 \mathrm{H}), 1.23$ (t, $J=$ $7.1 \mathrm{~Hz}, 3 \mathrm{H}), 1.17 \sim 1.03(\mathrm{~m}, 3 \mathrm{H}) ;{ }^{13} \mathrm{C} \mathrm{NMR}(101 \mathrm{MHz}$, $\left.\mathrm{CDCl}_{3}\right) \delta: 169.9,152.8,142.4,141.4,128.4,128.1,125.6$, $125.4,124.6,124.1,122.7,122.7,77.0,76.6,76.3,62.5$, 60.2, 59.5, 40.3, 13.9, 13.5; IR (neat) $v: 3067,2938,1735$, 1589, 1563, 1474, $763 \mathrm{~cm}^{-1}$. HRMS (ESI-TOF) calcd for $\mathrm{C}_{20} \mathrm{H}_{23} \mathrm{~N}_{2} \mathrm{O}_{3}\left([\mathrm{M}+\mathrm{H}]^{+}\right)$339.1709, found 339.1707.

乙基-2-(2-乙氧基-3-环已基-3,4-二氢喹唑啉-4-基) 乙酸酯(4b): 黄色油状物. ${ }^{1} \mathrm{H}$ NMR (400 $\left.\mathrm{MHz}, \mathrm{CDCl}_{3}\right) \delta$ : $7.20 \sim 7.13(\mathrm{~m}, 1 \mathrm{H}), 7.02(\mathrm{ddd}, J=20.3,7.7,1.4 \mathrm{~Hz}, 2 \mathrm{H})$, $6.91(\mathrm{td}, J=7.4,1.3 \mathrm{~Hz}, 1 \mathrm{H}), 4.93(\mathrm{dd}, J=9.0,4.4 \mathrm{~Hz}$, $1 \mathrm{H}), 4.42 \sim 4.32(\mathrm{~m}, 2 \mathrm{H}), 4.03(\mathrm{qd}, J=7.1,2.6 \mathrm{~Hz}, 2 \mathrm{H})$, $3.80 \sim 3.66(\mathrm{~m}, 1 \mathrm{H}), 2.62(\mathrm{dd}, J=14.8,9.1 \mathrm{~Hz}, 1 \mathrm{H}), 2.47$ $(\mathrm{dd}, J=14.8,4.4 \mathrm{~Hz}, 1 \mathrm{H}), 1.99 \sim 1.92(\mathrm{~m}, 1 \mathrm{H}), 1.81$ (ddq, $J=18.2,12.7,2.7 \mathrm{~Hz}, 2 \mathrm{H}), 1.71 \sim 1.54(\mathrm{~m}, 4 \mathrm{H}), 1.36 \sim$ $1.27(\mathrm{~m}, 5 \mathrm{H}), 1.14(\mathrm{t}, J=7.2 \mathrm{~Hz}, 4 \mathrm{H}) ;{ }^{13} \mathrm{C}$ NMR $(101$ $\left.\mathrm{MHz}, \mathrm{CDCl}_{3}\right) \delta: 170.2,154.8,143.0,127.7,124.8,124.0$, $121.9,121.8,62.1,60.0,57.2,52.2,41.7,32.1,31.5,25.8$, 25.6, 24.9, 14.1, 13.6; IR (neat) v: 2978, 2930, 1728, 1597, 1585, 1561, 759, $722 \mathrm{~cm}^{-1}$. HRMS (ESI-TOF) calcd for $\mathrm{C}_{20} \mathrm{H}_{29} \mathrm{~N}_{2} \mathrm{O}_{3}\left([\mathrm{M}+\mathrm{H}]^{+}\right)$345.2178, found 345.2169.

辅助材料(Supporting Information) 化合物 3 和 4 的核 磁共振谱图。这些材料可以免费从本刊网站 (http://sioc-journal.cn/)上下载.

\section{References}

[1] (a) Cai, C.-J.; Hu, B.-C.; Lü, C.-X. Chin. J. Org. Chem. 2005, 25, 
1311.

(b) Zeng, X.-C.; Xu, S.-H.; Li, Y.-Q.; Shi, W.-B.; Deng, Q.-Y. Chin. J. Org. Chem. 2004, 24, 802.

(c) Zhao, L.; Liang, Z.-H.; Yuan, S.-G. J. Henan Chem. Ind. 2003, $12,12$.

(d) Zhang, J.; Fan, X.-D.; Liu, Y.-F.; Li, H. J. Modern Chem. Ind. 2006, 26, 47.

(e) Zhu, H.-Y.; Liu, F.-C.; He, H.-P. Chin. J. Appl. Chem. 1999, 16, 71.

(f) Chen, Y.-Q. Flavour Fragrance Cosmet. 1992, 12, 14 (in Chinese).

(陈煌强，香料香精化妆品, 1992, 12, 14.)

(g) Connolly, D. J.; Cusack, D.; O'Sullivan, T. P.; Guiry, P. J. Tetrahedron 2005, 61, 10153.

(h) He, L.; Li, H.; Chen, J.; Wu, X.-F. RSC Adv. 2014, 4, 12065.

[2] Zhang, W.-G.; Yang, L.-M.; Wang, W.-G.; Jiang, H.-J.; Xu, X.-Y. Chem. Reag. 1998, 20, 138 (in Chinese).

(张文官, 杨联明, 王文广, 江和金, 徐锡瑛, 化学试剂, 1998, 20, 138.)

[3] Ouyang, P.; Chen, G.; Zhang, X. Acta Petrolei Sinica 2013, 29, 447 (in Chinese).

(欧阳平，陈国需，张贤明，石油学报, 2013, 29, 447.)

[4] (a) Eynde, J. J.; Godin, J.; Mayence, A.; Maquestiau, A.; Anders, E. Synthesis 1993, 867.

(b) Maheswari, C. U.; Kumar, G. S.; Venkateshwar, M.; Kumar, R. A.; Kantam, M. L.; Reddy, K. R. Adv. Synth. Catal. 2010, 352, 341. (c) Zeng, Y.; Qiu, G.; Cai, L.; Pike, V. W. J. Heterocycl. Chem. 2010, 47, 1240.

[5] (a) Han, B.; Wang, C.; Han, R.-F.; Yu, W.; Duan, X.-Y.; Fang, R.; Yang, X.-L. Chem. Commun. 2011, 47, 7818.

(b) Yan, Y.; Wang, Z. Chem. Commun. 2011, 47, 9513. (c) Ju, J.; Hua, R.; Su, J. Tetrahedron 2012, 68, 9364.

[6] (a) Baker, B. R.; Almaula, P. I. J. Org. Chem. 1962, 27, 4672. (b) Abdel, R. J.; Voelter, W.; Saeed, M. Tetrahedron Lett. 2004, 45, 3475 .

(c) Zhou, J.; Fang, J. J. Org. Chem. 2011, 76, 7730.

(d) Niloofar, T.; Abolghasem, D. Chin. J. Chem. 2011, 29, 1685.

[7] (a) Hennequin, L. F.; Boyle, F. T.; Wardleworth, J. M.; Marsham, P. R. J. Med. Chem. 1996, 39, 695.

(b) Kalusa, A.; Chessum, N.; Jones, K. Tetrahedron Lett. 2008, 49, 5840 .

(c) Adib, M.; Ansari, S.; Mohammadi, A.; Bijanzadeh, H. R. Tetrahedron Lett. 2010, 51, 30.

[8] (a) Sun, S. F.; Chen, L.; Yang, X. H. Chin. J. Chem. 2011, $29,991$.

(b) Hirota, S.; Sakai,T.; Kitamura, N.; Kubokawa, K.; Kutsumura, N.; Otani, T.; Saito,T. Tetrahedron 2010, 66, 653.

(c) Wu, M. H.; Hu,J. H.; Shen, D. S.; Bremond, P.; Guo, H. B. Tetrahedron 2010, 66, 5112.

(d) Liang, Y.; He, H.-W.; Yang, Z.-W. J. Heterocycl. Chem. 2011, 48,88 .

(e) Zhong, Y.; Wang, L.; Ding, M-W. Tetrahedron 2011, 67, 3714.

[9] Larksarp, C.; Alper, H. J. Org. Chem. 2000, 65, 2773.

[10] (a) Zeng, F.-L.; Alper, H. Org. Lett. 2010, 12, 1188.

(b) Zeng, F.-L.; Alper, H. Org. Lett. 2010, 12, 3642.

[11] (a) Qiu, G.-Y.-S.; He, Y.-H.; Wu, J. Chem. Commun. 2012, 48, 3836.

(b) Qiu, G.-Y.-S.; Lu, Y.; Wu, J. Org. Biomol. Chem. 2013, 11, 798.

[12] Zhu, T.-H.; Wang, S.-Y.; Tao, Y.-Q.; Ji, S.-J. Org. Lett. 2015, 17, 1974.

[13] (a) Chen, T.; Xu, X.-P.; Ji, S.-J. J. Comb. Chem. 2010, 12, 659.

(b) Chen, T.; Xu, X.-P.; Ji, S.-J. J. Heterocycl. Chem. 2013, 50, 244.

(c) Chen, T.; Xu, X.-P.; Ji, S.-J. Tetrahedron 2011, 30, 5469.

(Cheng, F.) 